\title{
Streptococcus pyogenes infects human endometrium by limiting the innate immune response
}

\author{
Antonin Weckel, ${ }^{1,2}$ Thomas Guilbert, ${ }^{1}$ Clara Lambert, ${ }^{1,2}$ Céline Plainvert,, ${ }^{1,2,3,4}$ François Goffinet, ${ }^{2,5,6}$ Claire Poyart, ${ }^{1,2,3,4}$ \\ Céline Méhats, ${ }^{1,2}$ and Agnès Fouet ${ }^{1,2,3}$
}

'Université de Paris, Institut Cochin, INSERM, U1016, CNRS, UMR8104, Paris, France. Département Hospitalo-Universitaire Risk \& Pregnancy, Port Royal Maternity, Paris, France. ${ }^{3}$ Centre National de Référence des Streptocoques, ${ }^{4}$ Hôpitaux Universitaires Paris Centre, Cochin, Assistance Publique Hôpitaux de Paris, ${ }^{5}$ Faculté de Médecine, Université Paris Descartes, and ${ }^{6}$ Service de Gynécologie Obstétrique I, Maternité Port Royal, Assistance Publique-Hôpitaux de Paris, Paris, France.

Group A Streptococcus (GAS), a Gram-positive human-specific pathogen, yields 517,000 deaths annually worldwide, including 163,000 due to invasive infections and among them puerperal fever. Before efficient prophylactic measures were introduced, the mortality rate for mothers during childbirth was approximately $10 \%$; puerperal fever still accounts for over 75,000 maternal deaths annually. Yet, little is known regarding the factors and mechanisms of CAS invasion and establishment in postpartum infection. We characterized the early steps of infection in an ex vivo infection model of the human decidua, the puerperal fever portal of entry. Coordinate analysis of CAS behavior and the immune response led us to demonstrate that (a) GAS growth was stimulated by tissue products; (b) CAS invaded tissue and killed approximately $50 \%$ of host cells within 2 hours, and these processes required SpeB protease and streptolysin 0 (SLO) activities, respectively; and (c) GAS impaired the tissue immune response. Immune impairment occurred both at the RNA level, with only partial induction of the innate immune response, and protein level, in an SLO- and SpeB-dependent manner. Our study indicates that efficient CAS invasion of the decidua and the restricted host immune response favored its propensity to develop rapid invasive infections in a gynecological-obstetrical context.

\section{Introduction}

Puerperal fever currently accounts for more than 75,000 maternal deaths annually. The Gram-positive human-specific pathogen Streptococcus pyogenes, also known as group A Streptococcus (GAS), is responsible for the most severe forms of infection (1). Between 2010 and 2016, GAS was responsible for over 50 and 100 postpartum infections per 100,000 persons per year in the United States and the United Kingdom, respectively $(2,3)$. In France in 20172018 , every year an average of $60 \%$ of GAS invasive infections occurred in the gyneco-obstetrical sphere for women between 18 and 40 years of age. In addition to puerperal fever, GAS causes a wide range of infections, from noninvasive infections, such as pharyngitis and impetigo, to invasive infections, such as necrotizing fasciitis, bacteremia, and streptococcic toxic shock syndrome, altogether responsible for 163,000 deaths annually (4).

GAS is genetically diverse (5). Strain genotyping relies on the sequence of the $5^{\prime}$ end of the $\mathrm{emm}$ gene and more than $200 \mathrm{emm}$ types exist (6). emm 28 strains are associated with obstetrical and gynecological infections and the emm 28 type is among the 3 most prevalent emm types involved in invasive infections in Europe (7-9). Virulence factors contributing to invasive diseases, including the

Authorship note: CM and AF contributed equally to this work.

Conflict of interest: The authors have declared that no conflict of interest exists.

Copyright: (5) 2021, American Society for Clinical Investigation.

Submitted: June 3, 2019; Accepted: December 10, 2020; Published: February 15, 2021.

Reference information: J Clin Invest. 2021;131(4):e130746.

https://doi.org/10.1172/JCl130746. extracellular cysteine protease SpeB and cytotoxin streptolysin $\mathrm{O}$ (SLO), have been described in vitro and confirmed in vivo in animal models of infection (10-12). SpeB cleaves many host molecules including matrix proteins; its in vivo importance has been inferred from its prevalence and demonstrated by in vivo experiments (1315). SLO, in addition to being a pore-forming toxin, facilitates the entry of the NAD-glycohydrolase into eukaryotic cells and together these toxins contribute to cell lysis and to GAS pathogenesis $(16,17)$.

In contrast with commensals or other pathogens, GAS colonizes only rarely the vagina of healthy individuals (0.03\%; ref. 18). Yet, before the introduction of efficient hygienic prophylactic measures, up to $10 \%$ of postpartum women died of puerperal fever. Furthermore, half of the cases of GAS-related puerperal fever occur within the first 2 days postpartum, with rapid progression to severe sepsis (19). We hypothesized that GAS efficiently and promptly infects the decidua, which is the mucosal uterine lining during pregnancy. This tissue is composed of roughly $60 \%$ endometrial decidualized stromal cells and $40 \%$ resident leucocytes (20). The immunological status of the decidua at the time of term birth is unique as compared with earlier terms, with higher abundance of T cells, macrophages, and dendritic cells and a decline in the NK cell number $(20,21)$. We recently showed that GAS readily adheres to primary human decidual stromal cells (22). However, to our knowledge, the early steps of human decidua infection by GAS or more generally, of GAS human tissue invasion, have not been characterized.

The current model describing the early steps of GAS invasive infections suggests adhesion to host tissue, growth, and invasion of the tissue within 24 hours postinfection (hpi) (23). These steps 
are followed by resistance to immune responses, tissue destruction, and systemic GAS infection (24). The observations of necrotizing fasciitis biopsy support the clinical relevance of these results (23). However, questions remain regarding the events occurring during the first hours of tissue infection, particularly when GAS reaches nonepithelial tissues such as the decidua.

In this work, we investigated the early events of GAS infection, and identified factors leading to the rapid progression of infection. To that end, we developed an integrated experimental model that simultaneously studies diverse aspects of the infection including the bacterial outcome and the host immune response. We show that GAS multiplies at the decidual surface and benefits from tissue-secreted products to do so, that it invades the tissue in the first 8 hours of infection, and that it kills half of the cells within 2 hours. SpeB and SLO, 2 major virulence factors, are implicated in these early infection steps. The host tissue immune response is restricted during these first infection steps.

\section{Results}

GAS grows on human decidua during the early steps of infection. We used 2 complementary approaches to follow tissue colonization and invasion: static and flow conditions (Figure 1). Human decidual explants were infected within hours of surgery with a wild-type (WT) isolate or its derivative expressing GFP (GFP-WT). When static conditions were used fixation was carried out at different time points; flow conditions were used for live microscopy. The decidua is composed of a fibronectin layer that covers the cells which are embedded in an extracellular matrix containing type IV collagen (Figure 1A, Supplemental Figure 1, and Supplemental Figure 3B; supplemental material available online with this article; https:/doi. org/10.1172/JCI130746DS1). GAS efficiently adheres to the tissue surface. Furthermore, in flow and static conditions, GAS-covered surface or the thickness of bacterial microcolonies increased in a time-dependent fashion, indicating the successful tissue surface colonization by GAS (11 out of 11 tissues) (Figure 1, Supplemental Figure 2, and Supplemental Table 1). GAS was observed to cover the entire tissue surface (Figure $1 \mathrm{C}$ and Supplemental Video 1). We precisely quantified GAS multiplication at the tissue surface during the first hours after infection in flow conditions (Figure 1D and Supplemental Table 1). GAS grew exponentially at the tissue surface, with doubling of the colonized surface every 82 minutes (Figure 1 , $\mathrm{E}$ and $\mathrm{F}$, and Supplemental Video 2). Determination of the thickness of bacterial microcolonies further showed that the mean value doubled in 4 hours, reaching a mean value of $4.2 \mu \mathrm{m}$ (Figure 1, G and $\mathrm{H})$. However, GAS thickness was locally heterogeneous, and at $6 \mathrm{hpi}$ reached $10 \mu \mathrm{m}$ in some hot spots and up to $16 \mu \mathrm{m}$ at $24 \mathrm{hpi}$ (Figure $1 \mathrm{G}$ and data not shown). Yet, the overall thickness increase was similar in all tissue samples considered (Figure 1, G and $\mathrm{H}$ ). Hence, growth occurs in all 3 dimensions and GAS doubling time in contact with the decidua is close to 60 minutes; this accounts for GAS's robust capacity to infect human decidua.

To analyze if diffusible host factors promoted GAS growth, we compared GAS growth in RPMI medium and in tissueconditioned supernatants from 8 samples (Supplemental Table 1 and Figure 1I). Whereas GAS does not multiply in RPMI, it multiplied in some conditioned supernatants (Supplemental Table 1 and Figure 1I). In contrast with the tissues, not all conditioned supernatants elicited growth; GAS grew in 5 out of the 8 samples. However, both tissues with supernatants that did not elicit growth and for which surface colonization was analyzed proved to support GAS multiplication (Supplemental Table 1). Since women were excluded if prescription antibiotics were used during the 2 weeks preceding delivery, except for preoperatively administered cephalosporin, and tissues were all similarly extensively rinsed in PBS, we excluded a role for antibiotic interference in GAS growth in supernatants. We therefore considered that nutrients secreted by uninfected tissues and bactericidal molecules might impact GAS growth. Antimicrobial peptides (AMPs), including human $\beta$ defensins (hBD1 and -2) and LL-37, were previously detected in the decidua during pregnancy (25). To determine whether their presence could explain GAS growth limitation, we measured the concentrations of these AMPs in conditioned supernatants (Figure 1J). No significant differences were detected between tissue samples, indicating that AMP concentrations are not predictive of GAS growth potential in tissue supernatants (Figure 1I). That GAS multiplies on all tested tissues but only in some conditioned supernatants needs to be further investigated.

GAS invades human decidua ex vivo. We then characterized GAS penetration of the tissue. Although GAS is nonmotile, bacteria were found below the tissue surface as soon as 4 hpi in static conditions (Supplemental Figure 3A). We quantified invasion events using automated image analysis (Figure 2, A and B, and Supplemental Figure 3B). As soon as $4 \mathrm{hpi}$, we observed an average of 600 invasion events per $\mathrm{mm}^{2}$; this corresponds to approximately 2 invading chains per 1000 surface chains. Furthermore, there were 3 times more intratissular bacteria at 8 hpi than at 4 hpi (Figure 2C). At 16 hpi, GAS were observed in direct contact with and inside stromal cells (Supplemental Figure 3C). We hypothesized that GAS invasion is an active process that is at least partly due to tissue degradation, especially fibronectin degradation, which is the upper layer of the tissue (Figure 1). SpeB, the major GAS protease, which is expressed in vivo and degrades fibronectin (26), may be involved in tissue penetration. To test this hypothesis, we infected decidua with either the WT or a $\Delta$ SpeB strain. At $4 \mathrm{hpi}$, the efficiency of tissue invasion of the $\Delta$ SpeB strain was approximately $50 \%$ of that of the WT strain, indicating that $\mathrm{SpeB}$ or products derived from SpeB activity contribute to the initial tissue invasion step (Figure 2C).

Another potential source of bacterial invasion is internalization into host motile cells. We observed phagocytosis of GAS by immune cells, presumably macrophages given the fairly large size of the cells, 15 minutes after infection in flow conditions. Intracellular bacteria were localized $6 \mu \mathrm{m}$ below the tissue surface for at least 4 hpi (Figure 2, D and E, Supplemental Figure 3D), indicating that GAS may invade the decidua as intracellular bacteria. Our results indicate that GAS can rapidly and actively invade the decidua and may exploit survival in decidual immune cells.

GAS induces cell death. GAS strains are cytotoxic to immune or epithelial cells in vitro. The factors responsible for toxicity may vary with GAS emm type (27). However, whether GAS cytotoxicity is involved in the early infection steps, particularly in decidua invasion, remains largely undocumented. We thus analyzed the consequences of ex vivo GAS infection of the human decidua on the physical state of cells in static conditions. As early as $4 \mathrm{hpi}$, we observed cell blebbing and dying immune cells, as indicated 
A

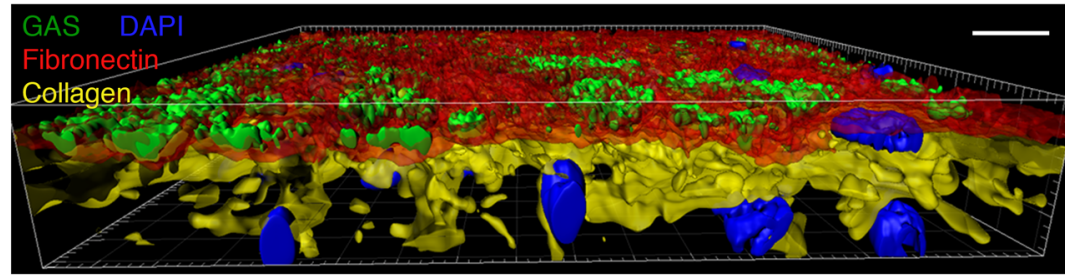

C
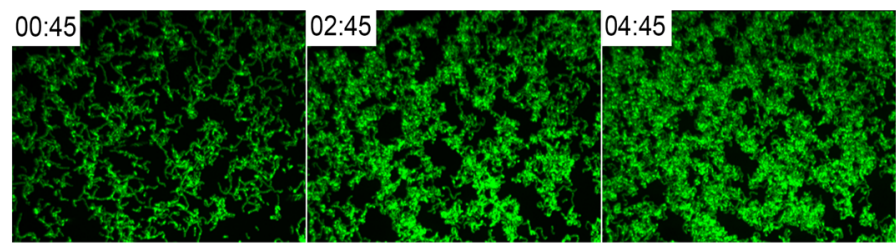

|06:45
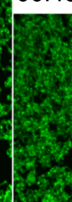

E
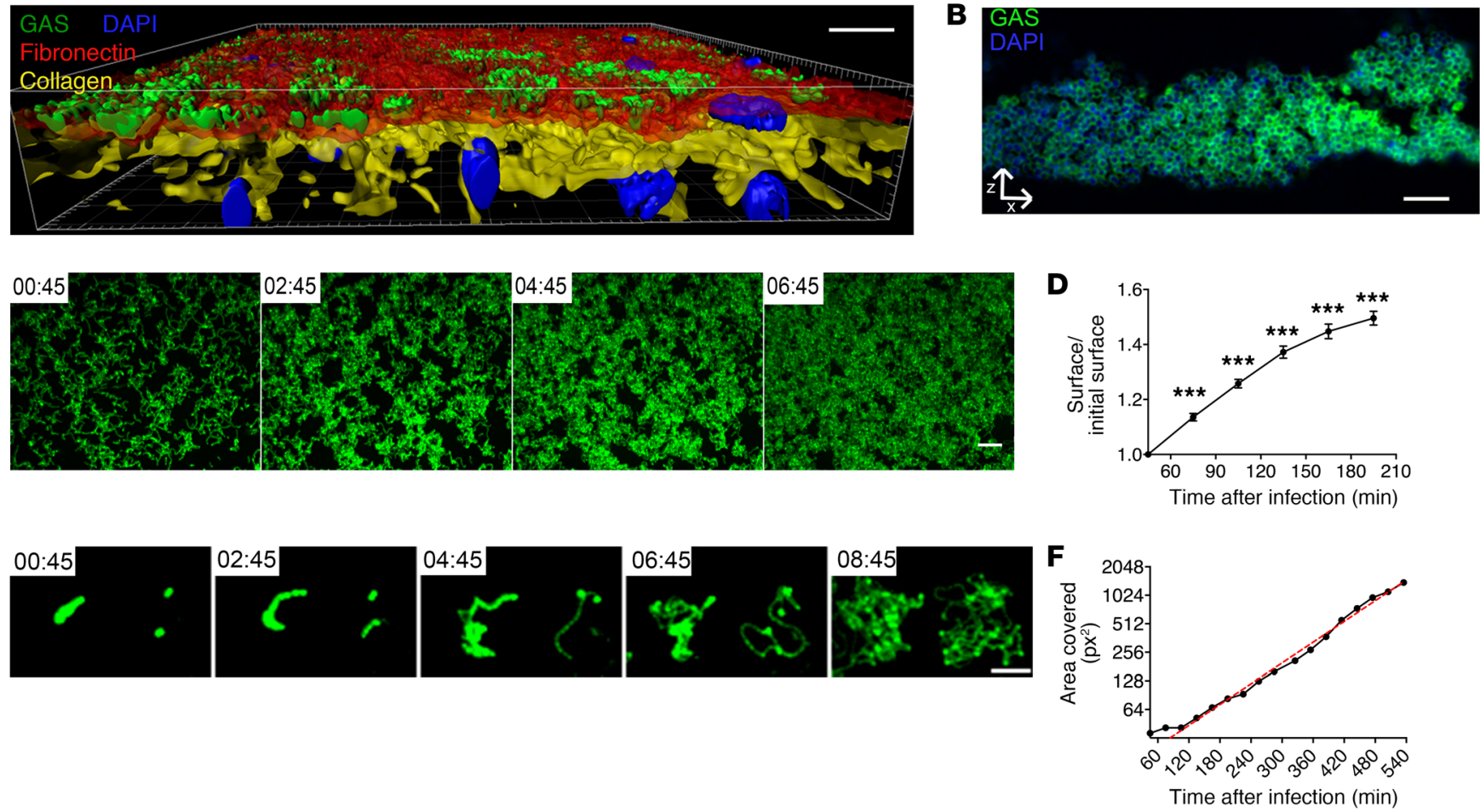

G

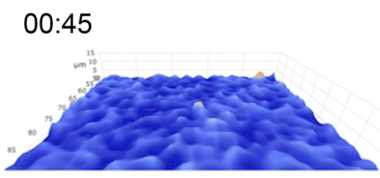

$02: 15$

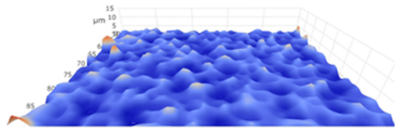

$03: 45$
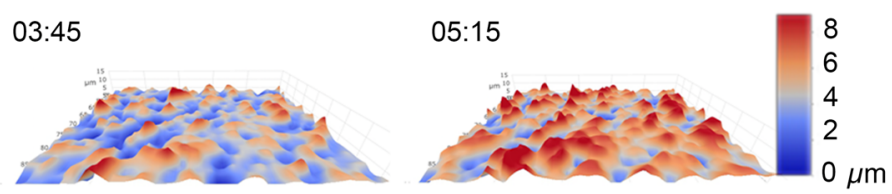

H

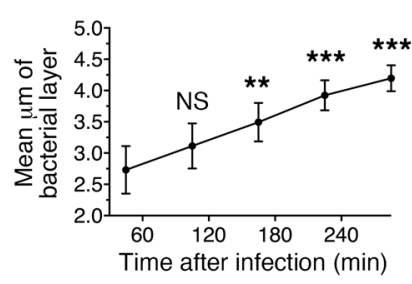

I

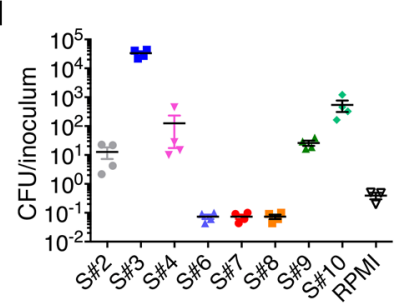

J

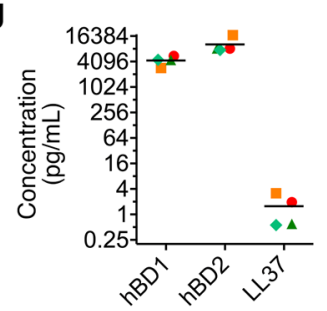

Figure 1. GAS adheres to the decidua and grows on it using tissue-secreted products. (A) Imaris 3D representation of a tissue infected 16 hours and imaged en face. Fibronectin, red; type IV collagen, yellow; GFP-GAS, green; DAPI, blue. Scale bar: $20 \mu \mathrm{m}$. Magnification: $\times 40$. (B) Immunofluorescence of a paraffin-embedded tissue transverse slice (24 hpi). Anti-GAS, green; DAPI, blue. Scale bar: $10 \mu \mathrm{m}$. Magnification: $\times 100$. (C and E) z-Max intensity projections of GFP signals from live en face acquisition images of GFP-WT at the tissue surface, at indicated time points (hr:min). Scale bars: $10 \mu \mathrm{m}$ (C) and $20 \mu \mathrm{m}$ (E). Magnification: $\times 25$. (D) Ratio over time of the area covered by WT GFP-CAS signal area in tissues from 5 samples including those in C. Mean values of 3 to 11 fields for each of the 5 tissues are plotted. (F) Quantification of the surface covered by GFP-GAS from the image in $\mathbf{E}$. Red dotted line, exponential curve fitted starting at 105 minutes; $R^{2}=0.9948$. (G) 3D surface heatmap of the bacterial layer thickness at different time points (hr:min) (live imaging). The $x, y$, and $z$ axes are scaled, color code in micrometers. Image size: $303 \times 190 \mu \mathrm{m}$. (H) Quantification of bacterial layer mean thickness; mean values of 2 to 13 fields for each of 5 tissues are plotted. (I) Multiplication factor after GAS incubation for 8 hours in tissue-conditioned medium or RPMI. $\mathrm{S \#}$ indicates the sample number. (J) Basal levels of accumulation of the indicated antimicrobial peptide in the supernatant of noninfected decidual tissues 8 hours after addition of fresh RPMI. Symbols are the same as in panel I. For all panels, error bars are standard error of the mean. ${ }^{* *} P<0.005,{ }^{* *} P<$ 0.001 by 2-way ANOVA at the initial time points ( $\mathbf{D}$ and $\mathbf{H})$.

by $\mathrm{CD} 45^{+}$membranes and DAPI staining (Figure 3A and Supplemental Video 3). At 16 hpi, a TUNEL assay indicated the presence of nuclei with altered morphology and DNA damage (Figure $3 \mathrm{~B})$; moreover, all cell types, immune $\left(\mathrm{CD} 45^{+}\right)$and nonimmune $\left(\mathrm{CD} 45^{-}\right)$, were damaged (Figure 3C). GAS-induced cytotoxicity increased with time after infection; $27 \%$ and $57 \%$ of cells were TUNEL positive at $2 \mathrm{hpi}$ and $8 \mathrm{hpi}$, respectively, compared with
$7 \%$ and $11 \%$ without infection (Figure 3, D and E). To determine if cell death was induced by a GAS-secreted factor, we measured cytotoxicity when bacteria were physically separated from the tissue via a Transwell insert (Figure 3E). In these conditions, GAS was still able to induce $21 \%$ and $37 \%$ of cell death at 2 hpi and 8 hpi, respectively, which demonstrates that GAS-secreted factors induce cell death without bacterial contact of host cells (at a 
A
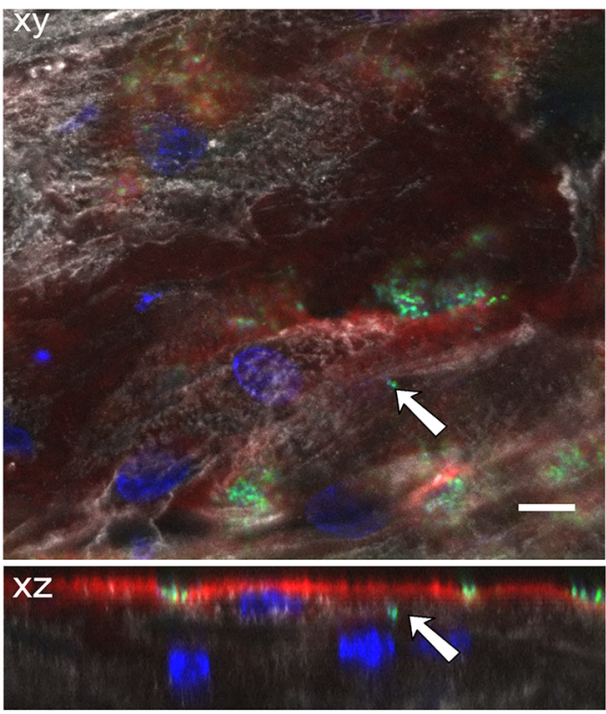

Fibronectin

Collagen

GAS

DAPI
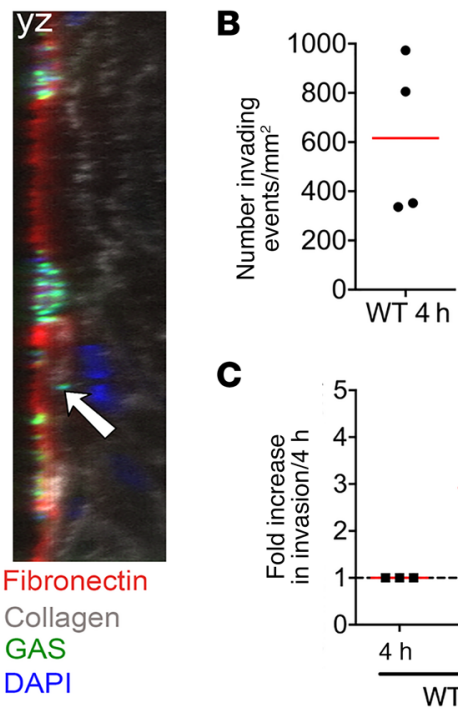

C

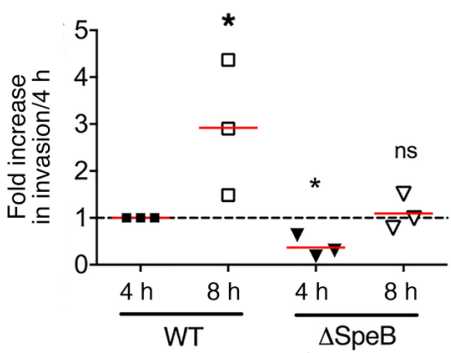

D
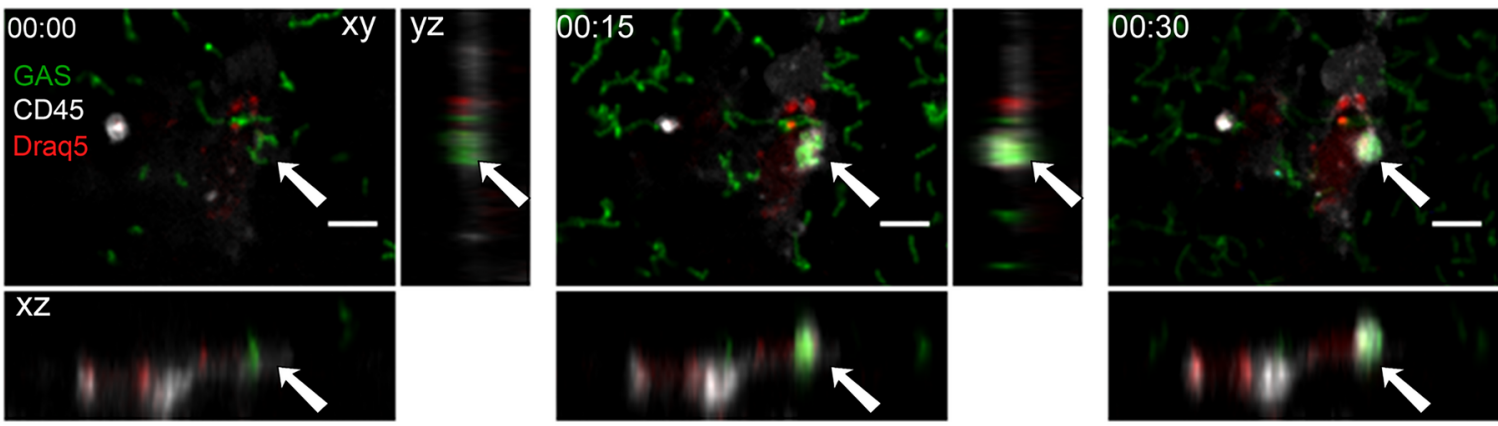

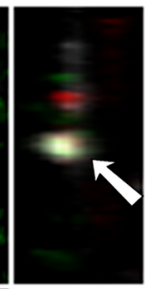

E
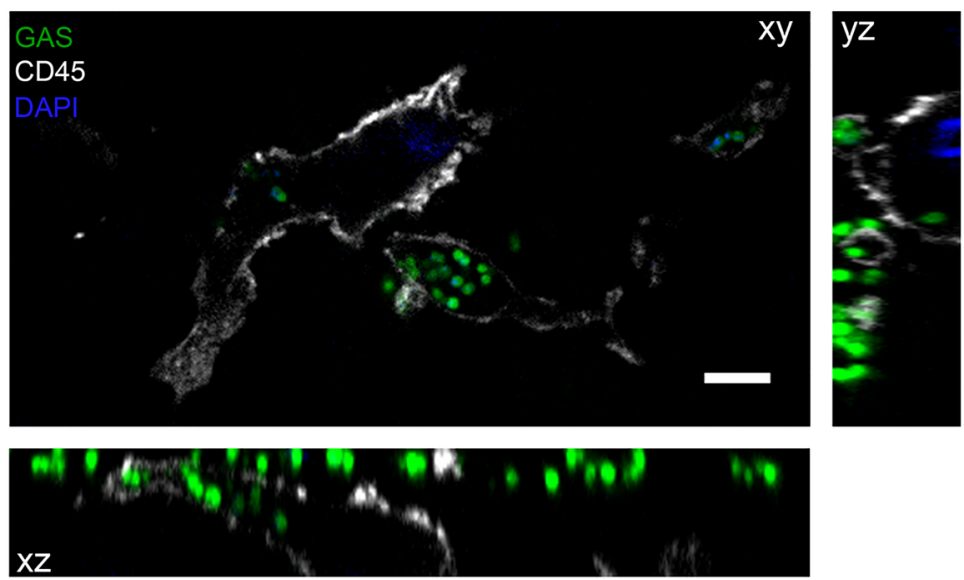

Figure 2. GAS actively invades the decidual tissue. (A) Orthogonal view of a tissue $16 \mathrm{hpi}$, static conditions; see also Supplemental Figure 3B. Arrows indicate an invading particle. Fibronectin, red; type IV collagen, gray; GAS, green; DAPI, blue. Scale bar: $10 \mu \mathrm{m}$. Magnification: $\times 40$. (B) Mean number of invading events per $\mathrm{mm}^{2}$ at $4 \mathrm{hpi}$ under static conditions with the GFP-WT strain calculated using high-throughput image analysis. Four samples are shown. (C) Ratio of GFP-WT and GFP- $\Delta$ SpeB invading events 4 and 8 hpi under static conditions. ${ }^{*} P<0.05$ by 1 -sample $t$ test vs. the WT at 4 hpi for WT 8 hpi and $\Delta$ SpeB $4 \mathrm{hpi}$, and vs. $\Delta$ SpeB 4 hpi for $\Delta$ SpeB $8 \mathrm{hpi}(n=3)$. (D) Time-lapse (hr:min), flow conditions, of an immune cell phagocytosing GAS in situ, with orthogonal view. Anti-CD45 (immune cells), gray; CAS, green; DRAQ5 (DNA), red. White arrows indicate the localization of the phagocytosis event. Scale bar: $10 \mu \mathrm{m}$. Magnification: $\times 25$. (E) Orthogonal views of GFP-WT bacteria inside the tissue and within an immune cell; image taken $6 \mu \mathrm{m}$ under the tissue surface 3 hpi under flow conditions; see also Supplemental Figure 3D. Anti-CD45, gray; bacteria, green; DAPI, blue. Scale bar: $5 \mu \mathrm{m}$. Magnification: $\times 100$.

distance $>250 \mu \mathrm{m})$. As these values are lower than when bacteria are in contact with tissues, it is possible that direct contact or proximity increases cytotoxicity efficiency.

SLO is a pore-forming toxin described to induce cell death (28, 29). We tested to what extent SLO was involved in GAS cytotoxicity in primary decidual stromal cells, and in whole decidua, at 2 and $4 \mathrm{hpi}$ (Figure 3, F and G). In primary decidual stromal cells at $4 \mathrm{hpi}$, the WT and isogenic revertant (BTSLO) strains induced $60 \%$ and $54 \%$ cytotoxicity. In striking contrast, the isogenic SLO-deleted mutant strain $(\triangle \mathrm{SLO})$ induced only $14 \%$ cytotoxicity (Figure $3 \mathrm{~F}$ ). In comparison, 
A
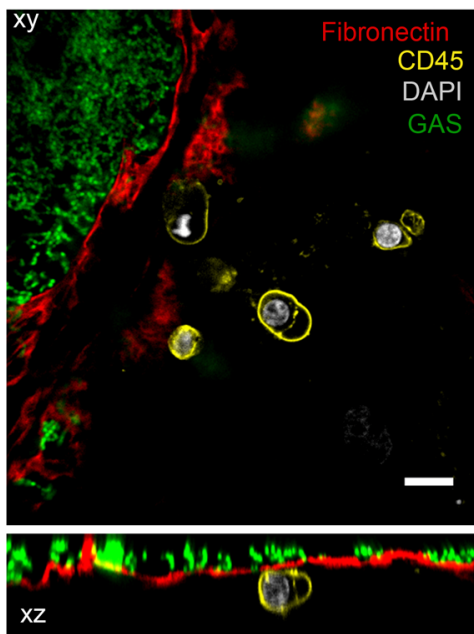

C
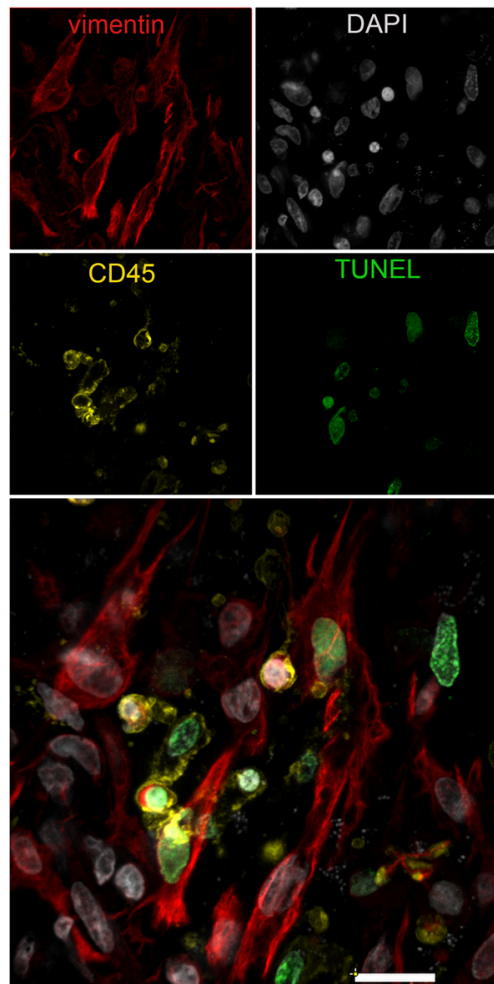

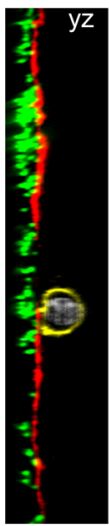

D
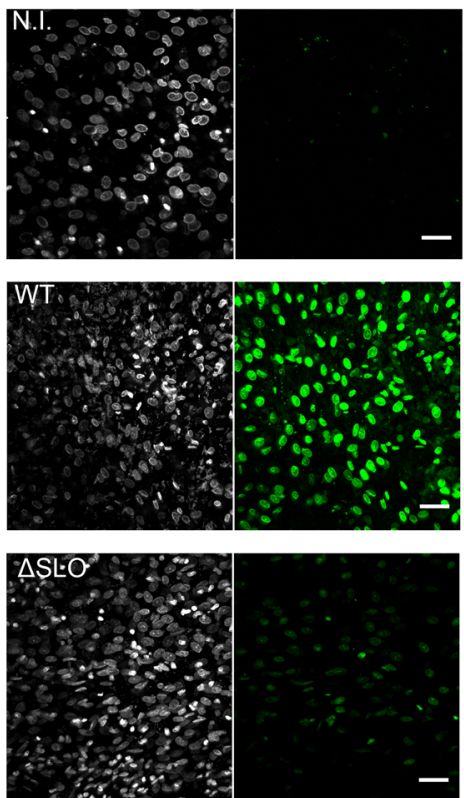

DAPI

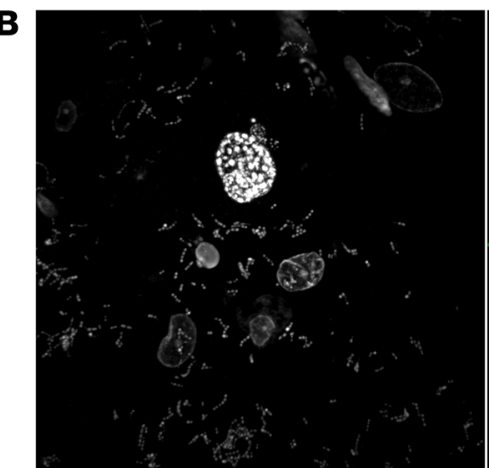

DAPI

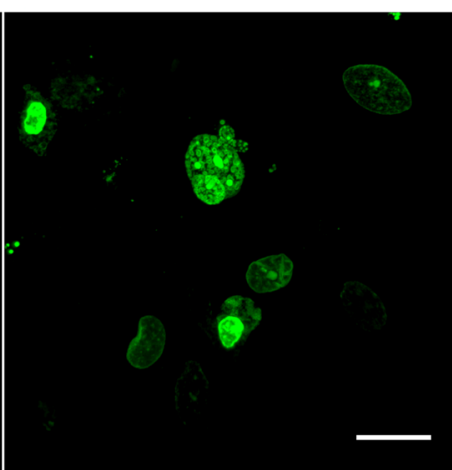

TUNEL

E

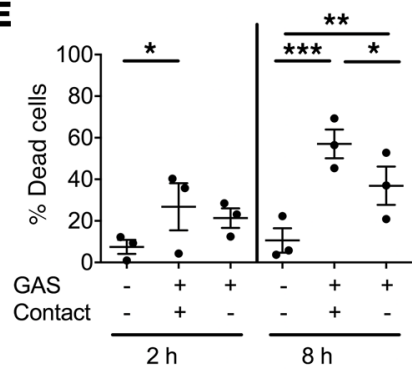

$\mathbf{F}$

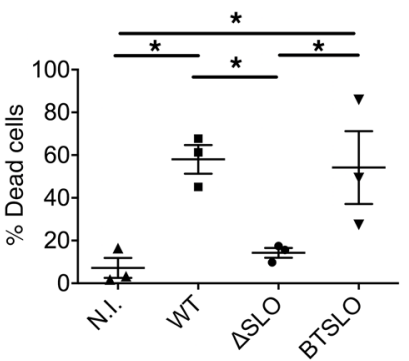

$\mathbf{G}$

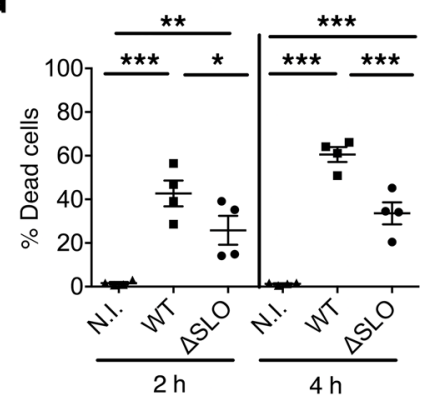

Figure 3. GAS induces stromal and immune cell death via processes involving SLO and other secreted factors. (A) Orthogonal view of a sample 5 hpi under static conditions prestained with anti-fibronectin (red), anti-CD45 (yellow), WT-GFP bacteria (green), and DAPI (gray). Scale bar: $10 \mu \mathrm{m}$. Magnification: $\times 40$. (B) Immunofluorescence of the decidua 16 hpi under static conditions. DAPI, gray; TUNEL, green. Scale bar: $10 \mu \mathrm{m}$. Magnification: $\times 63$. (C) Immunofluorescence of a sample $16 \mathrm{hpi}$ under static conditions. Stromal cells are vimentin ${ }^{+} \mathrm{CD} 45^{-}$and immune cells are CD45. Vimentin, red; DAPI, gray; CD45, yellow; TUNEL, green. Scale bar: $10 \mu \mathrm{m}$. Magnification: $\times 40$. (D) Immunofluorescence of a sample $4 \mathrm{hpi}$ under static conditions with the indicated strains. N.I., not infected. DAPI, gray; TUNEL, green. Scale bar: $40 \mu \mathrm{m}$. Magnification: $\times 20$. (E-G) Quantification of cytotoxicity by determination of the percentage of dead cells (E) on decidua with 3 samples, in the absence, contact (+), or the presence, contact (-), of a Transwell insert at indicated times; (F) on primary decidual cells from 3 different samples with indicated strains $4 \mathrm{hpi}$; and (G) on decidua with 4 samples with indicated strains, at indicated times. ${ }^{*} P<$ 0.05 ; ${ }^{*} P<0.005 ;{ }^{* *} P<0.001$ by 2-way ANOVA with Bonferroni's post hoc test (E and $\mathbf{G}$ ) or paired 1-way ANOVA $(\mathbf{F})$.

cytotoxicity in control noninfected cells was 7\%. These results confirm the involvement of SLO in the GAS cytotoxic effect. In decidual tissue, the $\triangle$ SLO strain was approximately $50 \%$ as cytotoxic as the WT strain ( $25 \%$ vs. $43 \%$ at 2 hpi, and $33 \%$ vs. $60 \%$ at 4 hpi, respectively; Figure $3 \mathrm{G})$. However, at both time points, the $\Delta$ SLO strain still induced significant cytotoxicity compared with the noninfected control (1.6\% and $1.4 \%$ at 2 and $4 \mathrm{hpi}$, respectively), indicating that GAS produces cytotoxic molecules in addition to SLO. In conclusion, GAS yields dramatic cell damages within a few hours of infection of the decidua, and SLO has an important role in toxicity. 


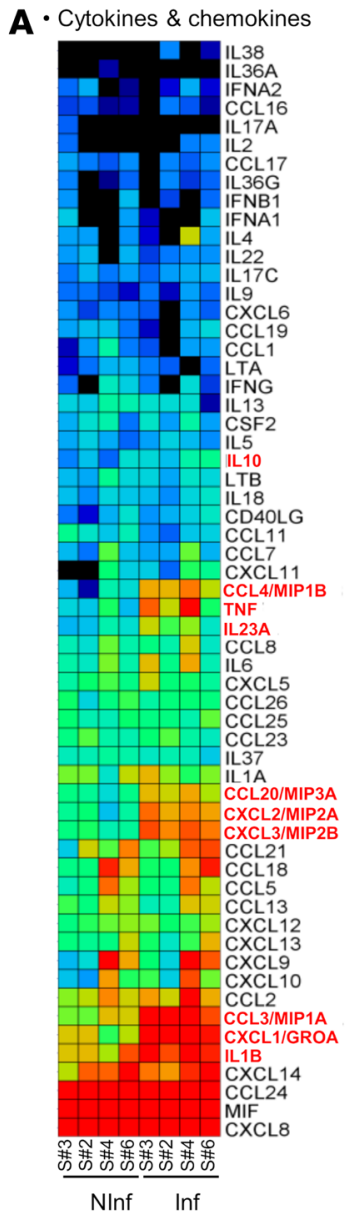

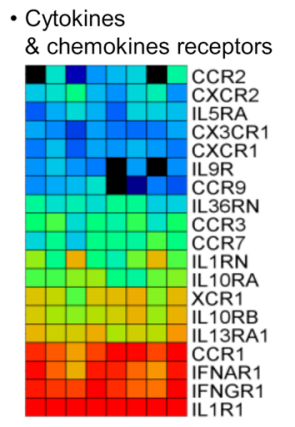
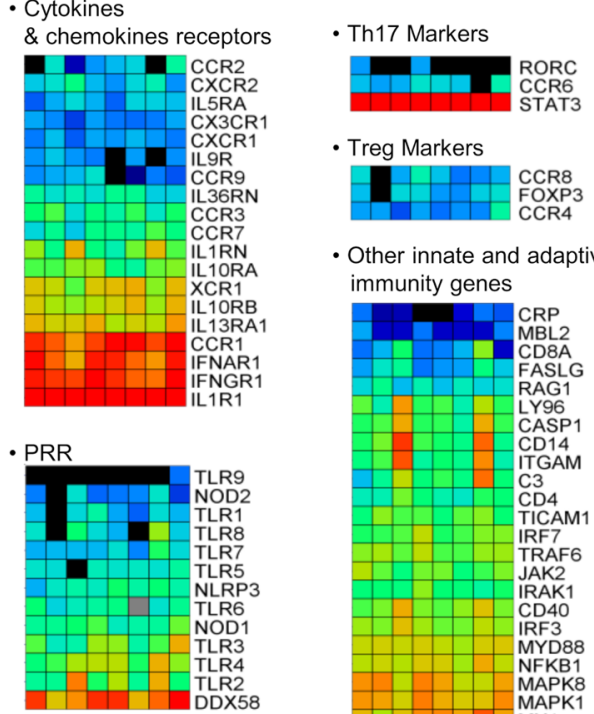

- Th1 Markers

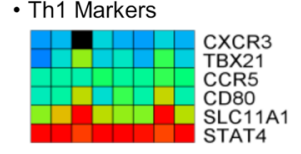

- Other innate and adaptive immunity genes
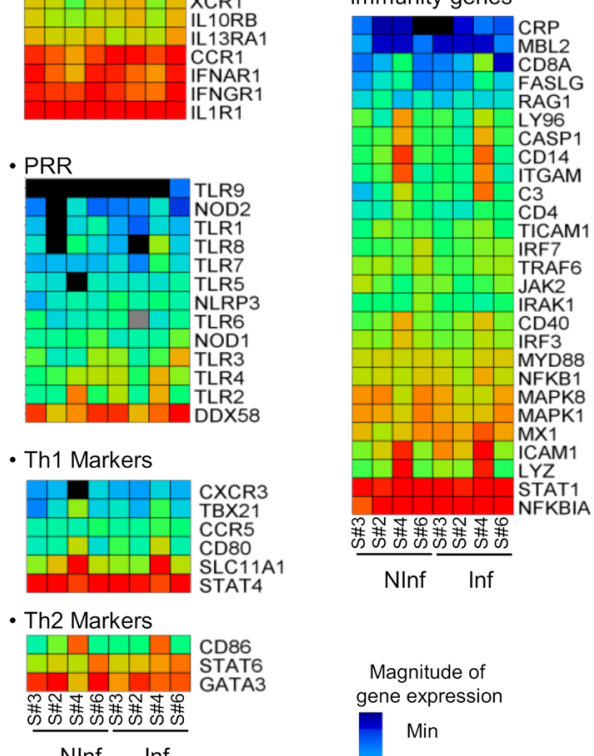

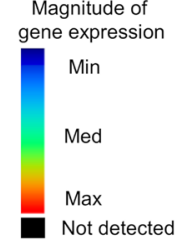

Figure 4. Host cytokine responses to GAS infection. (A) Heatmap of the results from the qRT-PCR screening using Qiagen $\mathrm{RT}^{2}$ Profiler PCR Arrays "Inflammatory cytokines and receptors" and "Human innate $\&$ adaptive response" on 4 samples, noninfected (NInf) or infected 8 hours under static conditions with the WT strain (Inf). Cytokines significantly overexpressed in the infected compared with noninfected condition are shown in red. (B and $\mathbf{C}$ ) Fold-change variation of the expression of the immune-related genes (B) 4 hpi (left) and $8 \mathrm{hpi}$ (right) compared with the noninfected expression. ${ }^{a} P<0.05$ compared with noninfected by 1-way Mann-Whitney $U$ test. (C) The concentration of the indicated cytokines compared with the noninfected condition (left, 4 hpi; right, 8 hpi) from 4 different samples. ${ }^{a} P<0.05$ compared with noninfected; ${ }^{b} P<0.05$ compared with WT; Friedman's test with Dunn's post hoc multiple-comparison test. Axes in $\mathbf{B}$ and $\mathbf{C}$ are $\log _{2}$ transformed. Symbols are as in Figure 1l. The infecting strain, $\mathrm{WT}, \Delta \mathrm{SLO}$, or $\Delta \mathrm{SpeB}$ is indicated below the $x$ axis. Means are indicated with black lines. Dotted lines correspond to a ratio of 1 .

B

$4 \mathrm{~h}$

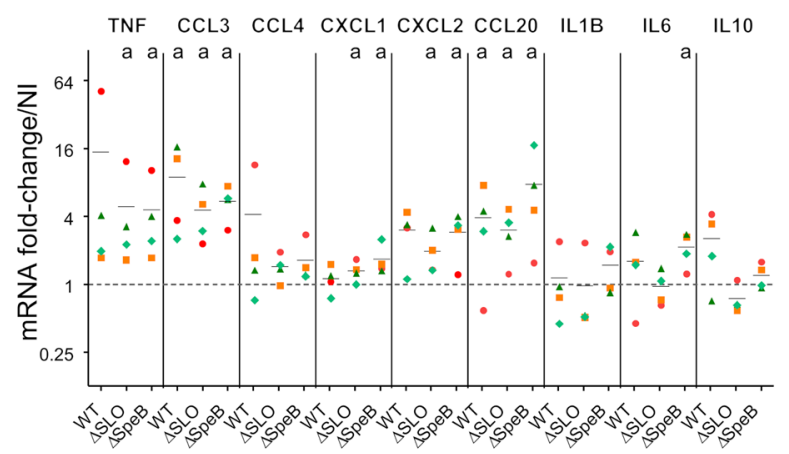

C

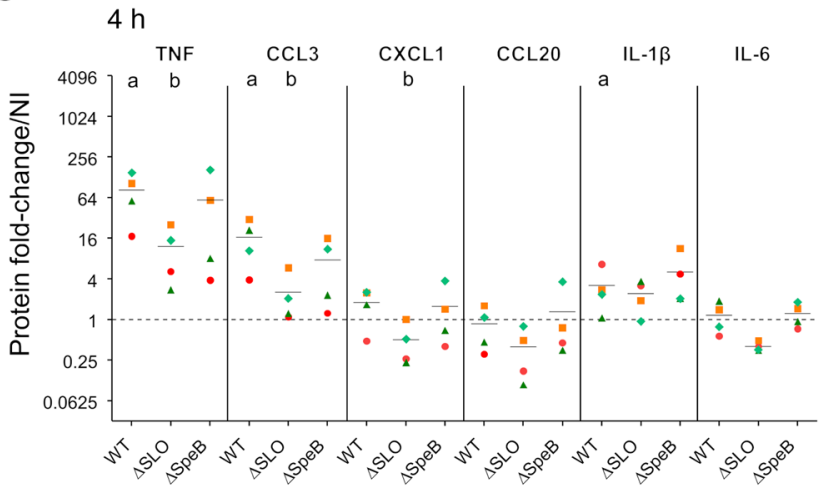

$8 \mathrm{~h}$

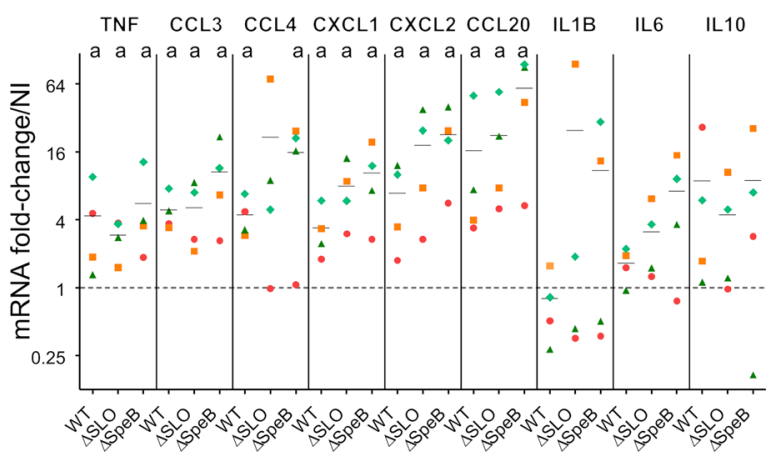

$8 \mathrm{~h}$

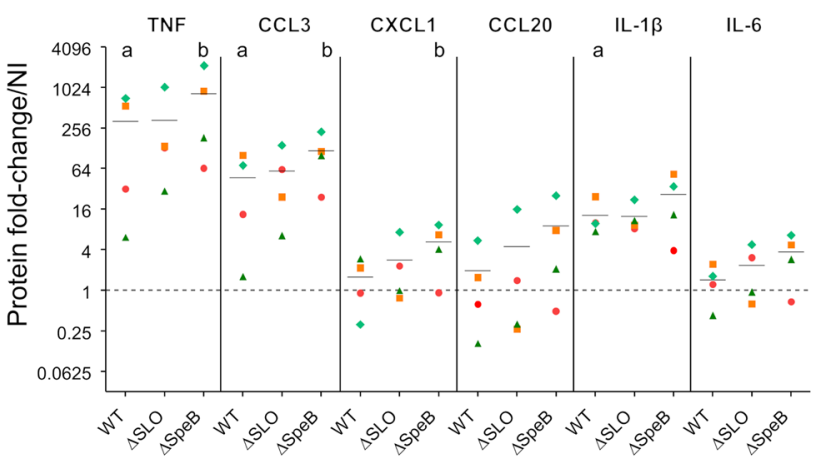




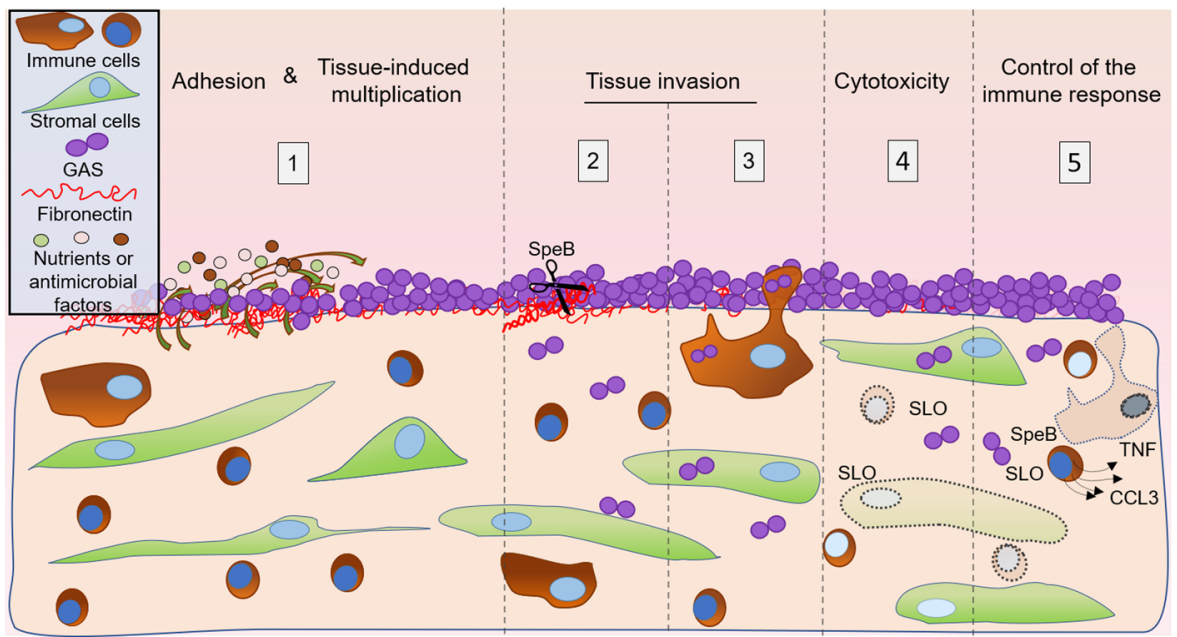

Figure 5. Schematic representation of the initial steps of decidual invasive infection by GAS. The successive steps are represented. 1: The decidua secretes nutrients and antimicrobial peptides (Figure 11), promoting GAS multiplication at the tissue surface (Figure 1, C-H). 2: GAS invades the tissue in, among others, an SpeB-dependent manner, most likely through its capacity to degrade fibronectin (Figure 2, A-C). 3: GAS is phagocytosed at the surface and is present inside decidual immune cells at least $6 \mu \mathrm{m}$ below the surface and $4 \mathrm{hpi}$, suggesting CAS may use immune decidual cells to invade the decidua (Figure 2, D and E). 4: GAS induces the death of decidual stromal and immune cells in an SLO-dependent mechanism (Figure 3). 5: GAS limits the innate immune response, impairing the response amplification (Figure 4). Decidual immune and stromal cells are represented as observed in the tissue; curved arrows = nutrients, AMP, or growth signaling factor secretion and diffusion; dotted line objects = damaged cells; dotted arrows = cytokine secretion .

results show that GAS specifically induces a low and limited inflammation at $8 \mathrm{hpi}$.

We performed qRT-PCR on 4 other uninfected and GAS-infected tissues at 4 hpi and 8 hpi to confirm the changes in gene expression. Expression was followed for 8 of the 10 genes overexpressed after GAS infection, as well as IL-6, the classical proinflammatory cytokine, the expression of which did not appear modified in the arrays (Figure 4B and Supplemental Figure 4A). Overexpression of 2 genes, encoding CCL3 and CCL20, occurred as soon as $4 \mathrm{hpi}$; at $8 \mathrm{hpi}$ the expression of TNF, CCL4, CXCL1, and CXCL2 was also increased (Figure 4B). Overexpression of IL-10 was not statistically different in this set of tissues. However, an analysis of the 8 samples together indicated a statistically different overexpression of IL-10 (fold-change $5.45 \pm 3.07, P=0.0156$ ). This suggests that IL-10 is induced by GAS infection. Notably, qRT-PCR assays confirmed that IL-6 is not significantly overexpressed after GAS infection, in contrast with its observed overexpression with GBS at 8 hpi (Supplemental Table 2 and ref. 33).

GAS limits the tissue inflammatory response. Efficient GAS multiplication in immune decidual cells led us to question whether the host mounted an inflammatory response to GAS. Expression was first analyzed for 133 unique immune genes related to inflammatory cytokines and receptors, and human innate and adaptive response pathways, using 2 commercial RT-PCR arrays, in noninfected decidua. This analysis provided the steady-state levels of immune response factors (Figure 4). As expected, the majority of the genes tested showed low to moderate expression, as compared with housekeeping genes (Figure 4A, Supplemental Table 1 , and ref. 30). Infection with GAS led to the induction of only 10 genes at $8 \mathrm{hpi}$. Nine genes encode cytokines and chemokines related to acute inflammatory response, including TNF, CXCL1, CXCL2, and IL-23A that are characteristic of an immediateearly-gene primary response (Figure 4A, Supplemental Table 2, and refs. 31, 32). The tenth gene encodes the antiinflammatory cytokine IL-10. This expression pattern suggested that either ex vivo decidua is unable to mount an immune response or that GAS infection restrains this response. The low induction level of GAS contrasts with much higher induction levels by group B Streptococcus (GBS) in a similar setup of infection (33). GBS infection modified the expression of 32 out of these 133 genes, and furthermore, the most activated genes compared with uninfected tissue are related to cellular response to cytokine stimulus (33). This supports the capacity of the decidua to elicit an immune response. Notably, in contrast with GBS, GAS infection fails to induce certain secondary response genes, such as NFKB1, CCR7, CD80, and ICAM1. This comparison indicates that GAS may impair the robust immune response in tissue that is elicited by GBS, a Streptococcus that elicits milder endometritis (34). Altogether, these
As GAS also modulates the in vitro accumulation of cytokines at the protein level (35-37), we asked whether the overexpression of 6 cytokine genes correlated with an increase in protein concentrations in tissue supernatants (Figure $4 \mathrm{C}$ and Supplemental Figure $4 \mathrm{~B})$. The basal level of accumulation of the 6 cytokines in the uninfected tissues 4 hpi and 8 hpi had the same pattern, independently of the sample, with high concentrations of IL-6 and CXCL2 (Supplemental Figure 4B and Supplemental Tables 3 and 4), known to be highly expressed in different subsets of decidual cells during pregnancy (30). After infection, the levels of TNF, CCL3, and IL-1 $\beta$ were higher as early as $4 \mathrm{hpi}$ and further increased at 8 hpi (Figure $4 \mathrm{C}$ and Supplemental Tables 3 and 4). However, overexpression of the CXCL2 gene at $4 \mathrm{hpi}$ and of the CXCL2 and CCL20 genes at 8 hpi (Figure 4B) were not paralleled by an increase in the amounts of these 2 proteins (Figure 4C), suggesting that GAS may control the immune response at a posttranscriptional level.

Modulation of the immune response might be a key step during GAS invasive infection, and appears to be controlled on transcriptional and posttranscriptional levels. We assessed the potential roles of SLO and SpeB on that limitation in the decidua $(35,38)$. Transcriptional activation of the tested genes was similar in the WT strain and the $\triangle \mathrm{SLO}$ and $\triangle \mathrm{SpeB}$ mutant strains (Figure $4 \mathrm{~B})$. Remarkably, accumulation of some cytokines was different after infection with the mutant strains. The SLO-deficient strain showed reduced accumulation of TNF, CCL3, CXCL2, and IL-6 at $4 \mathrm{hpi}$ (Figure $4 \mathrm{C}$ ). The $\Delta \mathrm{SpeB}$ mutant induced a cytokine accumulation comparable to that of the WT at 4 hpi. However, accumulation of TNF, CCL3, and CXCL2 was significantly greater in the $\Delta$ SpeB- than in the WT-infected condition at $8 \mathrm{hpi}$ (Figure $4 \mathrm{C})$. This indicates that SLO is, at least in part, responsible for 
the accumulation of these cytokines, whereas the production of SpeB, directly or indirectly, increases GAS's capacity to inhibit cytokine accumulation.

These results suggest that the moderate accumulation of cytokines after GAS infection is the result of a fine balance between pro- and antiinflammatory responses induced by GAS. Also, during GAS's efficient colonization and invasion of the human decidua, SLO accentuates the inflammatory response while SpeB attenuates it.

\section{Discussion}

GAS is established as the major etiological agent of puerperal fever and maternal death. A key element of GAS infection is the rapid onset, i.e., development of fever within 2 days following delivery. To bypass the roadblock brought by GAS human specificity, studies of GAS noninvasive and invasive infections, and host responses during emm1-elicited pharyngitis were conducted in nonhuman primates $(14,39-42)$. However, these models do not permit direct visualization of colonization nor of early invasion steps. We overcame this limitation by using the original conjunction of ex vivo infection of human decidua, bacterial genetic manipulation, state-of-the-art imaging, and immune response analyses. Our main findings are that (a) GAS adheres and grows on human decidual tissue within the first hours of infection; (b) GAS actively kills cells and invades this tissue, a process that requires SLO and SpeB, respectively; and (c) GAS limits the host immune response (Figure 5). The ex vivo setup developed here enabled us to dissect GAS's capacity to establish decidua infection in real time and to simulate an in vivo situation.

We observed significant GAS growth; to our knowledge, our study provides the first estimate of GAS doubling time in contact with a human tissue, roughly 60 minutes; it corresponds to a $10^{7}$ multiplication in 24 hours, and could account for the rapidity with which puerperal fever occurs. Our results indicate that the decidua secretes nutrients and/or growth signaling molecules (Figure 5). The secreted nutrient(s)/growth signal(s) may be numerous. That in some cases GAS grows on a tissue but not in the corresponding conditioned supernatant suggests that compounds may be secreted in response to infection or that cell lysis releases nutrients. Also, growth factors are present in several conditioned supernatants and are most likely not asparagine, which is used by GAS for sensing and proliferation (43). In our study, the 15 amino acids for which GAS is auxotrophic are supplied in the medium (44). Also, the described mechanism required bacteria-host cell contact that is absent when preparing conditioned supernatants. Furthermore, GAS grows on all tissues and some conditioned supernatants and dies in other supernatants, suggesting that secreted nutrients and/or bactericidal products may differ between individuals, even before infection. A difference in quantities of the human defensins or AMPs did not explain a difference in growth. Indeed, hBD1 and hBD2 concentrations were identical in all tissues. As for LL-37, its concentration varied between individuals, but this would probably have few consequences for GAS killing since SpeB inactivates LL-37 at the bacterial surface, representing a resistance mechanism (45).

Invasion initiation on human decidua by GAS has not been previously documented to the best of our knowledge (Figure 5).
Our findings show that factors produced by GAS actively promote its penetration into human tissue. SpeB has broad proteolytic activity, including the degradation of extracellular matrix proteins; its role in tissue invasion may be linked to this capacity (13). Yet, considering the pleiotropic effects of the absence of SpeB on the GAS surfactome and on GAS virulence mechanisms (13), including the modified SLO production in the $\triangle$ SpeB strain, our data do not exclude a role for other virulence factors in the decreased invasion of the $\Delta$ SpeB mutant strain. As the decidua parietalis is not covered by epithelial cells, bacteria that cross the extracellular matrix are in direct contact with stromal and immune cells, among which neutrophils are scarce (46). GAS at the cell surface readily invaded phagocytic cells in the first minutes of infection and were still present at 4 hpi (Figure 5). GAScharged phagocytes may facilitate penetration of decidua in agreement with previous reports (47-49).

GAS induces the death of about half the stromal and immune cells in the decidua (Figure 5). The $\triangle$ SLO mutant strain is less cytotoxic than the WT strain, supporting in vitro-obtained conclusions (50). Moreover, the $\triangle$ SLO mutant strain still induced cell death, suggesting the cytotoxic action of other GAS-secreted products, such as streptolysin S (51). Hence, GAS cytotoxicity could account for the failure of cells to clear GAS infection (52).

Dense macrophage and neutrophil infiltrations are observed during established GAS severe invasive soft tissue infections in humans (48), which would be absent in ex vivo infection models (52). However, as seen upon GBS infection, such a setup does allow assessment of IL- 6 and CXCL8 production by tissue cells, which attracts neutrophils (53). The ex vivo setup is thus a key readout for the in vivo situation. In our study, GAS jeopardized the upregulation of inflammatory mediators for neutrophil and macrophage recruitment (Figure 5). Also, the GAS-specific concomitant absence of signaling mediators, especially from the $\mathrm{NF}-\kappa \mathrm{B}$ family, indicates that GAS limits the decidua innate response, including the response amplification. Our results regarding GAS's ability to prevent the activation of the immune response support and expand the conclusion that cytokine production is decreased during an experimental pharyngeal infection of nonhuman primates (40). GAS is less common than GBS in the female reproductive tract, but typically causes more severe maternal infection. In a comparable study, GBS did not readily cross healthy-term maternal-fetal membranes (54). Altogether, these data indicate that limited inflammation may favor GAS infection initiation by delaying or decreasing the recruitment of immune cells. These responses may account for the GAS-puerperal fever association.

The integrated model, comprising an in situ setup and multiple analyses developed in this study, offers a valuable tool to study bacterial tissue invasion and toxicity, which is applicable to various invasive pathogens and tissue types. Other GAS virulence factors implicated via in vitro studies in nonimmune cell entry, phagocytosis, and inflammation modulation could be tested by our holistic approach $(13,24)$. Transposon libraries could also be screened as a means of identifying novel colonization or invasion factors.

In conclusion, GAS actively multiplies, hides intracellularly, and diverts the host response during the very first hours following decidua infection. This rapidity may account both for the particu- 
lar capacity of GAS, despite its quasi-absence from the female genital tract microbiota, to elicit puerperal fever and for its swift establishment. Our elucidation of GAS infection of the human decidua supports epidemiological conclusions underlining the need for prophylactic measures $(2,55)$ and vaccine development to prevent life-threatening GAS infections such as puerperal fevers.

\section{Methods}

Strains and culture conditions. The strains used in this study are described in Supplemental Table 7. The M28PF1 strain is an emm28 clinical isolate responsible for an endometritis (French National Reference Center [CNR] for Streptococci; https://cnr-strep.fr/) that was selected on phenotypic and genotypic bases (56). More precisely, a collection of $50 \mathrm{emm} 28$ independent endometrial clinical isolates from different geographic locations and sampling years was constituted. For each strain, the presence of the specific RD2 sequence was tested, the sequence of the $\operatorname{cov} R, \operatorname{covS}$, and $\operatorname{rop} B$ genes, genes amenable to mutations in other emm types, was defined and the capacity to form a biofilm was measured (7, 57-59). A subgroup of $20 \mathrm{RD} 2^{+}$strains, WT for $\operatorname{cov} R, \operatorname{cov} S$, and $\operatorname{rop} B$ (since this was the most frequently observed genotype) and displaying an average biofilm formation capacity was constituted. To define isolates that possessed the same genes as most of the other $e m m 28$ endometrial isolates, the 20 strains were analyzed by DNA arrays covering the GAS pangenome. A subgroup of 8 representative isolates was then constituted and growth in THY supplemented with $20 \%$ human plasma, in the presence of cervical cells (HeLa), endometrial cells (HEC-1A), and pulmonary cells (A549), as well as their adhesion to these cell lines, were further analyzed. M28PF1 displays mean growth and adhesion values; therefore, it is a genetically and phenotypically representative emm 28 endometrial isolate. GAS strains were grown under static conditions at $37^{\circ} \mathrm{C}$ in Todd Hewitt broth supplemented with $0.2 \%$ yeast extract (THY) or on THY agar (THYA) plates. For GFP strains, the medium was supplemented with $10 \mu \mathrm{g} / \mathrm{mL}$ erythromycin. Prior to the infection, the bacterial solution was vortexed to disrupt bacterial cell chains.

Genetic constructions and generation of GFP-producing and SLOand SpeB-deleted mutant strains. The plasmids used in this study are described in Supplemental Table 7. The primers used for the generation of the plasmids and verifying the different strains are described in Supplemental Table 8. The strains harboring an integrated inducible Gfp gene were constructed as follows. We sequentially cloned into the $\mathrm{pG} 1$ vector (60) the Perm-Gfp transcriptional fusion surrounded by the KpnI and HindIII sites from pATGfp (61), giving rise to pG1Perm-Gfp. The lacA-lacR intergenic sequence, enabling a single cross-over in M28PF1 and derivatives, was amplified using the primers F_lacA and R_lacA and cloned by In-Fusion (Clontech) into pG1Perm-Gfp digested with EcoRI, giving rise to pG1-lacA-Perm-Gfp. The erm promoter was replaced by the tetO tetR Pxyl promoter region by amplifying it from pTCV_TetO (62) using the primers F_tetO and R_tetO and cloning it into pG1-lacA-Perm-Gfp previously digested with EcoRI-EcoRV, giving rise to pG1-lacA-PTetO-Gfp. The plasmid was checked by sequencing and transferred into M28PF1 or derivatives by electroporation as previously described (63). The correct localization of the construct was confirmed by sequencing the region using a primer outside the construct (R_extlacA) and one hybridizing to the vector, RP48. The uninduced GFP-producing strains grew like M28PF1 in laboratory THY. The construct was stable even in the absence of erythromycin for at least 12 generations. The anhydrotetracycline (Sigma-Aldrich) concentration required for full expression of GFP was $20-50 \mathrm{ng} / \mathrm{mL}$ and all bacteria were fluorescent after 90 minutes of induction; the fluorescence was stable for at least 6 hours when the bacteria were in the stationary growth phase. GFP fluorescence is observable when GAS is intracellular; induction of GFP does not affect strain growth.

The $\Delta$ SLO and $\triangle$ SpeB strains correspond to in-frame deletion mutants of the slo and speB genes and were obtained by homologous recombination of the plasmids pG1-SLO and pG1-SpeB following the same protocol as described previously $(22,64)$. The DNA fragments encompassing speB and slo were cloned in BamHI-EcoRI-digested pG1 using the In-Fusion cloning kit (Clontech). This led to deletion of nucleotides 72-1155 and 76-1690 for speB and slo, respectively. The back-to-the-WT (BTSLO and BTSpeB) strains were obtained during the second cross-over, which leads to the mutant or back to the WT strain. The strains were entirely sequenced and no spurious mutation were found. The absence of the cognate protein in the mutant strains was confirmed by Western blot or dot blot. The SLO and SpeB production in the $\Delta$ SpeB and $\Delta$ SLO strains, respectively, was assessed by Western blot or dot blot. The $\triangle \mathrm{SLO}$ strain produced similar amounts of SpeB as the WT strain. In contrast, the SLO production in the $\Delta$ SpeB strain was one-tenth of that of the WT.

For all experiments, GAS strains were prepared as follows. Overnight cultures were diluted to $\mathrm{OD}=0.05$ and grown in THY to the exponential phase $(\mathrm{OD}=0.5)$, centrifuged, and diluted in RPMI. For GFP expression, exponential-phase bacteria were further diluted to OD $=0.1$ in THY supplemented with $10 \mu \mathrm{g} / \mathrm{mL}$ erythromycin and $20 \mathrm{ng} / \mathrm{mL}$ anhydrotetracyclin, grown for 90 minutes at $37^{\circ} \mathrm{C}$, and diluted in RPMI.

Human tissue collection. Human placenta with attached maternalfetal membranes were collected from healthy women with an uncomplicated singleton pregnancy, undergoing a planned cesarean delivery prior to the onset of active labor at term (between 38 and 40 weeks of pregnancy). Except for per-operatively administered cephalosporin, women were excluded if prescription antibiotics were used during the 2 weeks preceding delivery. Supplemental Table 1 indicates in which experiment each sample tissue was used.

Maternal-fetal membrane processing. Within 15 minutes of collection, biological samples were processed in the laboratory. Maternalfetal membranes were detached from placenta under sterile conditions. All membranes were similarly extensively rinsed in PBS and carefully examined. Pieces of membrane that were not in the vicinity of either the placenta or the remodeled zone overlying the cervix (20), free of surface blood clots, were cut and glued to Petri dishes or coverslips with veterinary glue (3M Vetbond glue), with the fetal side sticking to the plastic or glass, and covered with RPMI.

In vitro bacterial growth measurement. Conditioned supernatants were prepared as follows. RPMI was added to maternal-fetal tissues for 8 hours at $37^{\circ} \mathrm{C}, 5 \% \mathrm{CO}_{2}$; the supernatants were subsequently filtered and diluted 1:5 with RPMI. Bacteria $(1000 / \mathrm{mL})$ were added to these diluted supernatants or to RPMI alone. After 8 hours of incubation at $37^{\circ} \mathrm{C}, 5 \% \mathrm{CO}_{2}$, solutions were serially diluted and plated. After 24 hours at $37^{\circ} \mathrm{C}, \mathrm{CFU}$ were counted.

Primary decidual stromal cell culture, infection, and analyses. Cells from 3 samples were isolated from the decidua parietalis obtained and processed as described above and cultured as previously 
described (22). One milliliter of GAS in RPMI at a bacterial density corresponding to an MOI of 100 was added to confluent decidual stromal cells for 4 hours at $37^{\circ} \mathrm{C}, 5 \% \mathrm{CO}_{2}$. Cells were extensively washed with PBS and fixed for 15 minutes at $20^{\circ} \mathrm{C}$ with $4 \%$ paraformaldehyde. Cytotoxicity was assessed using TUNEL staining following the manufacturer's protocol (DeadEnd Fluorometric TUNEL System, Promega) and a LEICA DMI 6000 microscope with a $20 \times$ objective (NA 0.5) for measurement.

Antibodies. The following antibodies were used during this study: Alexa Fluor 594 mouse anti-CD45 (Biolegend, clone M5E2), rabbit anti-fibronectin (Sigma-Aldrich, F3648), rabbit anti-vimentin (Abcam, ab92547), mouse anti-type IV collagen (DSHB, M3F7), and rabbit anti-whole GAS, gift from I. Julkunen (Department of Vaccination and Immune Protection, National Institute for Health and Welfare [THL], University of Helsinki, Finland). Secondary antibodies used were Alexa Fluor 594-conjugated anti-rabbit and Alexa Fluor 647conjugated anti-mouse antibodies (Invitrogen).

\section{Experiments in static conditions}

Immunofluorescence. Maternal-fetal membranes $\left(8 \mathrm{~cm}^{2}\right.$ or $\left.1.5 \mathrm{~cm}^{2}\right)$ were glued to a $35-\mathrm{mm}$-diameter Petri dish or on a 12-mm-diameter glass coverslip, respectively. When specified, tissues were prestained with anti-CD45 (1:100) for 30 minutes at $37^{\circ} \mathrm{C}, 5 \% \mathrm{CO}_{2}$. Tissues were infected with $0.2 \mathrm{~mL} / \mathrm{cm}^{2}$ of a solution of $1.7 \times 10^{8}$ bacteria $/ \mathrm{mL}$ in RPMI, supplemented with $10 \mu \mathrm{g} / \mathrm{mL}$ erythromycin and $50 \mathrm{ng} / \mathrm{mL}$ anhydrotetracycline (infection medium) when GFP strains were used. After infection, tissues were washed and fixed in formalin for 24 hours, and then stored in $70 \%$ ethanol at $4^{\circ} \mathrm{C}$ until use. Fixed tissues were further cut into $0.5 \mathrm{~cm}^{2}$ pieces and glued to coverslips, and permeabilized with $0.2 \%$ Triton $\mathrm{X}-100$ (Sigma-Aldrich) for 30 minutes at $20^{\circ} \mathrm{C}$. Blocking solution (PBS $+3 \% \mathrm{BSA}$ ) was added for 1 hour at $20^{\circ} \mathrm{C}$, and tissues were stained for 4 hours at $20^{\circ} \mathrm{C}$ with blocking solution containing primary antibodies: anti-GAS (1:250), anti-fibronectin (1:250), anti-type IV collagen (1:50), and anti-vimentin (1:250). Tissues were washed with PBS and secondary antibodies and DAPI (1:1000) in blocking solution were added for 1 hour at $20^{\circ} \mathrm{C}$.

Immunofluorescence was performed on paraffin-embedded tissues as described previously (65). Slices were cut (10 $\mu$ m thick) and stained with whole GAS rabbit antiserum (laboratory stock, 1:250).

TUNEL assay. RPMI $(500 \mu \mathrm{L})$ was added to $1.5 \mathrm{~cm}^{2}$ tissue glued to a 12-mm glass coverslip. One hundred microliters of M28PF1 at $5 \times$ $10^{8}$ bacteria/mL was added directly to the tissue or in the upper chamber of a $6.5 \mathrm{~mm}$ Transwell insert (polycarbonate, $0.4 \mu \mathrm{m}$ membrane, Costar). For the noninfected condition, $100 \mu \mathrm{L}$ of RPMI was added directly to the tissue. After the specified infection time, tissues were washed with PBS and fixed in formalin for 24 hours, and then stored in $70 \%$ ethanol at $4^{\circ} \mathrm{C}$ until use.

Slight modifications were brought to the TUNEL manufacturer's protocol. Pieces of tissue $\left(0.5 \mathrm{~cm}^{2}\right)$ were fixed and glued to coverslips and permeabilized with $500 \mu \mathrm{L}$ PBS with $0.2 \%$ Triton X-100 for 30 minutes at $20^{\circ} \mathrm{C}$. One hundred microliters of equilibration buffer (from the manufacturer's kit) was added to the tissues in a wet chamber for 15 minutes, after which the liquid was removed and $50 \mu \mathrm{L}$ of labeling solution was added for $3-4$ hours at $37^{\circ} \mathrm{C}$. Tissues were washed twice with saline-sodium citrate $2 \times$ buffer (from the manufacturer's kit) and stained with DAPI (1:2000) for 15 minutes. For double vimentin and TUNEL staining, TUNEL staining was performed first.
Static condition image acquisition. The maternal side of tissues was placed facing a 35-mm high glass-bottom $\mu$-dish (IBIDI) and imaged en face using a Yokogawa CSU-X1 Spinning Disk coupled with a Leica DMI6000B microscope. Acquisitions were made with MetaMorph 7 software (Molecular Devices). For measurement of surface coverage, the thickness of bacterial microcolonies, and quantification of bacterial invasion, images were acquired with a $40 \times$ objective (NA 1.25). For the latter, $0.3 \mu \mathrm{m} z$-steps and approximatively 90 slices per stack were acquired. For cytotoxicity quantification, images were acquired with a $20 \times$ objective (NA 0.7), $1 \mu \mathrm{m} z$-steps and for tissues approximatively 70 slices per stack were acquired.

Static condition image treatments and data analysis. All images were processed with Image (NIH). For cytotoxicity measurements, TUNEL- and DAPI-positive nuclei of images were segmented and automatically counted. At least 5 fields per condition were analyzed for tissues, and 10 fields for decidual stromal cells. To measure bacterial invasion, another ImageJ macro was used to segment bacterial particles on $z$-max projection images of GFP signals. For each bacterial particle, the axial localization was determined and compared to fibronectin and type IV collagen localization. Any bacterial particle whose signal was $1.2 \mu \mathrm{m}$ below fibronectin or collagen signals was considered as an invasion event. Five to 8 fields $(153 \times 169 \times 30 \mu \mathrm{m})$ per condition were analyzed. A GAS chain was considered to be on average 4 cocci, corresponding to the mean size of invading particles. We estimated the amount of invading chains by considering bacterial chain surface and thickness (mean thickness, $4 \mu \mathrm{m}$; bacterial coverage of a field, 90\%).

For local thickness measurement of the GFP signals corresponding to bacteria, the whole field was subdivided into regions of interest and a custom ImageJ macro measured the full width at half maximum (FWHM) of the $z$-axis GFP signal. The thickness value obtained by measuring the FWHM was then converted to micrometers by measuring the mean FWHM of 20 single bacteria, considered to be $1 \mu \mathrm{m}$ thick. Five to 7 fields per tissue were analyzed, with samples treated and acquired using the same procedure as for bacterial invasion. A 3D image representation of the tissue was performed using Imaris 7.4 (Bitplane AG).

\section{Experiments in flow conditions}

Maternal-fetal explants $\left(8 \mathrm{~cm}^{2}\right)$ were glued to a $35-\mathrm{mm}$ Petri dish and stained with DRAQ5 (1:2000) and anti-CD45 (1:100) in infection medium containing DAPI (1:30,000) (flow condition infection medium) for 30 minutes at $37^{\circ} \mathrm{C}, 5 \% \mathrm{CO}_{2}$. Tissues were extensively washed with flow condition infection medium and $3 \mathrm{~mL}$ of GFPproducing bacteria $\left(1 \times 10^{8} \mathrm{CFU} / \mathrm{mL}\right)$ in flow condition infection medium were added to the tissues in a $37^{\circ} \mathrm{C}$ microscopy chamber for 45 minutes until $20 \%-30 \%$ of the tissue surface was covered by bacteria. The supernatant was then discarded, tissue was washed with infection medium to remove unattached bacteria, and a peristaltic pump with circulating infection medium bubbled with $95 \% \mathrm{O}_{2}$ and $5 \% \mathrm{CO}_{2}$ was activated in an open circuit at $0.3 \mathrm{~mL} / \mathrm{min}$.

Flow condition image acquisition. Images of infected tissues were acquired en face with an upright DM6000FS Leica confocal microscope coupled with a Yokogawa CSU-X1 Spinning Disk, with a 25× objective (NA 0.95, Leica Microsystems). Acquisitions were made with MetaMorph 7 software and images were taken every 30 minutes for 3 hours with a $z$-step of $2 \mu \mathrm{m}$. For long-term acquisition, axial drift was manually compensated for after 3 hours and acquisition was resumed for another 3 hours. 
Flow condition image treatments and data analysis. All images were processed with ImageJ. 3D drifts of live images were corrected with custom routines. For surface area measurements of bacterial colonization, bacteria were segmented based on a threshold on the maximum intensity $z$-projection images of the GFP signal at the different time points, with the same threshold for each time point. Measurements of the local thickness was performed on images acquired in real time as described above for static images. Three to 12 fields per tissue were analyzed.

Bacterial layer thickness. A 3D surface heatmap was generated by a custom R code based on the plot-ly library.

Computer code availability. Computer codes used for this work will be transferred upon request.

RNA extraction. Static condition-obtained tissues were washed once and stored in TRI Reagent (Sigma-Aldrich) at $-80^{\circ} \mathrm{C}$ until used. Total RNA was isolated using a Qiagen RNeasy Kit according to the manufacturer's instructions. The purity and concentration of total RNA were evaluated using a NanoDrop spectrophotometer (Thermo Fisher Scientific), by measuring the absorbance at 260 $\mathrm{nm}$ and the ratio of absorbance at $260 \mathrm{~nm}$ and $280 \mathrm{~nm}$.

qRT-PCR analysis. RNAs were treated with deoxyribonuclease (Invitrogen, Life Technologies) to remove any contaminating DNA. Total RNA ( $4 \mu \mathrm{g})$ was reverse transcribed using random primers and M-MLV Reverse Transcriptase (Invitrogen), according to the manufacturer's instructions. Quantitative PCR was carried out on a Light Cycler 480, 96-well apparatus (Roche Diagnostics), with 160 ng of cDNA as a template, using the amplification kit SensiFAST SYBR No-Rox kit (Bioline), according to the manufacturer's instructions. The $\mathrm{RT}^{2}$ Profiler PCR Arrays (Qiagen) for inflammatory cytokines and receptors and human innate and adaptive response were performed according to the manufacturer's instructions. Gene expression was normalized using a panel of 5 housekeeping genes: ACTB, B2M, GAPDH, HPRT1, and RPLPO. $\triangle \mathrm{Ct}$ ranging from 0 to 6.0 was considered as the maximum magnitude of gene expression, from 6.1 to 9.9 as moderate, and from 10 to 15 as low. Genes with a nominal $P$ value $\leq 0.05$ were considered to be differentially expressed. Genes showing greater than 2 -fold variation were further considered in the analysis. Heatmaps were created using the MeV Package (http:// mev.tm4.org/). Enrichr (https://maayanlab.cloud/Enrichr/) was used for pathways enrichment analysis, restricted to Gene Ontology (GO) terms and ARCHS4 databases.

GBS infection of maternal-fetal membrane microarray subanalysis. Data were extracted using GEO2R from Park et al. (33). After conversion of the gene annotation using the DAVID gene ID Conversion tool (https://david.ncifcrf.gov/conversion.jsp), fold-changes and $P$ values for the 133 immune-related genes we previously analyzed with the $\mathrm{RT}^{2}$ Profiler Arrays were retrieved from 8-hour incubation samples (4 controls and 4 GBS-treated).

Protein and AMP analysis. Static condition-obtained supernatants of explants were stored at $-80^{\circ} \mathrm{C}$ until use. The levels of IL-6, TNF, CCL20, CXCL2, CCL3, and IL-1 were measured with a Bio-Plex custom assay (Bio-Rad) using a Bio-Plex 200. AMP concentrations were analyzed in the same supernatants by ELISA with the following kits: LL-37 (Hycult Biotech), hBD1 (R\&D Systems, Novus), and hBD2 (Elabscience). The concentrations are reported as $\mathrm{pg} / \mathrm{mL}$ medium. The samples were quantified in duplicate according to the manufacturers' instructions.

Statistics. Data were analyzed by Prism 6 software (GraphPad Software) or Xlstat version 2018.1 (Addinsoft). Where indicated, we used 2-way ANOVA. We used Student's $t$ test or nonparametric tests for quantitative variables as indicated in the text and Pearson's $\chi^{2}$ test for qualitative variables, as appropriate. A $P$ value of less than 0.05 was considered to be significant.

Study approval. The study of the human maternal-fetal membranes was approved by the local ethics committee (Comite de Protection des Personnes Ile de France III, no. Am5724-1-COL2991, 05/02/2013). All participants provided written informed consent prior to inclusion in the study at the Department of Obstetrics, Port Royal Maternity, Cochin University Hospital, Paris, France.

\section{Author contributions}

AW, TG, CL, CM, and AF designed experiments, carried out experiments and performed data analysis. C Plainvert, FG, and C Poyart provided essential reagents and scientific advice. $\mathrm{CM}$ and $\mathrm{AF}$ supervised the project. $\mathrm{AW}, \mathrm{CM}$, and $\mathrm{AF}$ wrote the manuscript.

\section{Acknowledgments}

We thank Louis Réot for the RT-PCR screening; Karine Bailly of Cochin Cytometry and Immunology (CYBIO) for the Luminex cytokine quantification; S. Brochet, Q. Cece, and M. Gaillard from AF and C Poyart's laboratory and T. Meylheuc from the Imaging Facility at the Microscopy and Imaging Platform (ISC MIMA2, INRAE, Jouy-en-Josas-Antony research center France, DOI: MIMA2, INRAE, 2018. Microscopy and Imaging Facility for Microbes, Animals and Foods, https://doi.org/10.15454/1.5 572348210007727E12) for assistance with in vitro experiments; E. Donnadieu, S. Barrin, E. Peranzoni, V. Feuillet, and A. Trautmann for advice in the imaging setup and helpful discussions; A. Gruss for helpful discussions and correcting the manuscript; I. Julkunen for the gift of anti-GAS antibodies; the Imag'IC facility of the Cochin Institute; and all the personnel of the CIC MèreEnfant Cochin-Necker. This work was supported by Université Paris Descartes (contract no. KL2UD to AW) the Département Hospitalo-Universitaire Risks in Pregnancy (PRIDE 2014, to AF and CM), INSERM, CNRS, Université de Paris, Centre National de Référence des Streptocoques.

Address correspondence to: Agnes Fouet, 22 rue Méchain, Paris 75014, France. Phone: 33.1.40.51.64.50; Email: agnes.fouet@ inserm.fr.

AW's current address: University of California, San Francisco, 513 Parnassus Ave, San Francisco, California 94143, USA.
1. Sriskandan S. Severe peripartum sepsis. JR Coll Physicians Edinb. 2011;41(4):339-346.

2. Leonard A, et al. Severe group A streptococcal infections in mothers and their newborns in London and the South East, 2010-2016: assess- ment of risk and audit of public health management. BJOG. 2019;126(1):44-53.

3. Deutscher $\mathrm{M}$, et al. Incidence and severity of invasive Streptococcus pneumoniae, group A Streptococcus, and group B Streptococcus infections among pregnant and postpartum women. Clin Infect Dis. 2011;53(2):114-123.

4. Carapetis JR, et al. The global burden of group

A streptococcal diseases. Lancet Infect Dis. 2005;5(11):685-694. 
5. Bessen DE, et al. Genetic correlates of throat and skin isolates of group A streptococci. J Infect Dis. 1996;173(4):896-900.

6. Beall B, et al. Sequencing emm-specific polymerase chain reaction products for routine and accurate typing of group A Streptococci. J Clin Microbiol. 1995;34(4):953-958.

7. Green NM et al. Genetic diversity among type emm28 group A Streptococcus strains causing invasive infections and pharyngitis. J Clin Microbiol. 2005;43(8):4083-4091.

8. Plainvert $\mathrm{C}$ et al. Invasive group A streptococcal infections in adults, France (2006-2010). Clin Microbiol Infect. 2012;18(7):702-710.

9. Gherardi G, et al. Prevalent emm types among invasive GAS in Europe and North America since year 2000. Front Public Health. 2018;6(3):59.

10. Hung $\mathrm{CH}$ et al. Synergistic effects of streptolysin $\mathrm{S}$ and streptococcal pyrogenic exotoxin $\mathrm{B}$ on the mouse model of group A streptococcal infection. Med Microbiol Immunol. 2012;201(3):357-369.

11. Limbago B, et al. Role of streptolysin O in a mouse model of invasive group A streptococcal disease. Infect Immun. 2000;68(11):6384-6390.

12. Walker MJ et al. Disease manifestations and pathogenic mechanisms of Group A Streptococcus. Clin Microbiol Rev. 2014;27(2):264-301.

13. Shannon BA, et al. Toxins and superantigens of Group A Streptococci. Microbiol Spectr. 2019;7(1):47-58.

14. Olsen RJ et al. The majority of 9,729 group A Streptococcus strains causing disease secrete SpeB cysteine protease: pathogenesis implications. Infect Immun. 2015;83(12):4750-4758.

15. Lukomski S et al. Inactivation of Streptococcus pyogenes extracellular cysteine protease significantly decreases mouse lethality of serotype M3 and M49 strains. JClin Invest. 1997;99(11):2574-2580.

16. Madden JC, et al. Cytolysin-mediated translocation (CMT): a functional equivalent of type III secretion in gram-positive bacteria. Cell. 2001;104(1):143-152.

17. Zhu L et al. Contribution of secreted NADase and Streptolysin O to the pathogenesis of epidemic serotype M1 Streptococcus pyogenes infections. Am J Pathol. 2017;187(3):605-613.

18. Mead PB, Winn WC. Vaginal-rectal colonization with group A streptococci in late pregnancy. Infect Dis Obstet Gynecol. 2000;8(5-6):217-219.

19. Hamilton SM, et al. Pregnancy-related group a streptococcal infections: Temporal relationships between bacterial acquisition, infection onset, clinical findings, and outcome. Clin Infect Dis. 2013;57(6):870-876.

20. Marcellin L, et al. Immune modifications in fetal membranes overlying the cervix precede parturition in humans. JImmunol. 2017;198(3):1345-1356.

21. Bartmann C, et al. Quantification of the predominant immune cell populations in decidua throughout human pregnancy. Am J Reprod Immunol. 2014;71(2):109-119.

22. Weckel A, et al. The N-terminal domain of the $\mathrm{R} 28$ protein promotes emm 28 group A Streptococcus adhesion to host cells via direct binding to three integrins. J Biol Chem. 2018;293(41):16006-16018.

23. Siemens N, et al. Biofilm in group A streptococcal necrotizing soft tissue infections. JCI Insight.
2016;1(10):151-157.

24. Cole JN, et al. Molecular insight into invasive group A streptococcal disease. Nat Rev Microbiol. 2011;9(10):724-736.

25. Yarbrough VL, et al. Antimicrobial peptides in the female reproductive tract: a critical component of the mucosal immune barrier with physiological and clinical implications. Hum Reprod Update. 2015;21(3):353-377.

26. Carroll RK, Musser JM. From transcription to activation: how group A Streptococcus, the flesh-eating pathogen, regulates SpeB cysteine protease production. Mol Microbiol. 2011;81(3):588-601.

27. Goldmann O, et al. Streptococcus pyogenes induces oncosis in macrophages through the activation of an inflammatory programmed cell death pathway. Cell Microbiol. 2009;11(1):138-155.

28. Bricker AL, et al. NAD ${ }^{+}$-glycohydrolase acts as an intracellular toxin to enhance the extracellular survival of group A streptococci. Mol Microbiol. 2002;44(1):257-269.

29. Timmer AM, et al. Streptolysin O promotes group A Streptococcus immune evasion by accelerated macrophage apoptosis. J Biol Chem. 2009;284(2):862-871.

30. Suryawanshi H, et al. A single-cell survey of the human first-trimester placenta and decidua. Sci Adv. 2018;4(10):eaau4788.

31. Goldfeld AE, et al. Transcription of the tumor necrosis factor alpha gene is rapidly induced by anti-immunoglobulin and blocked by cyclosporin A and FK506 in human B cells. Proc Natl Acad Sci U S A. 1992;89(24):12198-12201.

32. Smale ST. Selective transcription in response to an inflammatory stimulus. Cell. 2010;140(6):833-844.

33. Park H-R, et al. Group B Streptococcus activates transcriptomic pathways related to premature birth in human extraplacental membranes in vitro. Biol Reprod. 2018;98(3):396-407.

34. Cicinelli E, et al. Chronic endometritis due to common bacteria is prevalent in women with recurrent miscarriage as confirmed by improved pregnancy outcome after antibiotic treatment. Reprod Sci. 2014;21(5):640-647.

35. Egesten A, et al. SpeB of Streptococcus pyogenes differentially modulates antibacterial and receptor activating properties of human chemokines. PLoS One. 2009;4(3):1-9.

36. Zingaretti $C$, et al. Streptococcus pyogenes SpyCEP: a chemokine-inactivating protease with unique structural and biochemical features. FASEB J. 2010;24(8):2839-2848.

37. Klenk M, et al. Global epithelial cell transcriptional responses reveal Streptococcus pyogenes Fas regulator activity association with bacterial aggressiveness. Cell Microbiol. 2005;7(9):1237-1250.

38. Harder J, et al. Activation of the Nlrp3 inflammasome by Streptococcus pyogenes requires Streptolysin $\mathrm{O}$ and NF- $\kappa \mathrm{B}$ activation but proceeds independently of TLR signaling and P2X7 receptor. JImmunol. 2009;183(9):5823-5829.

39. Virtaneva K, et al. Longitudinal analysis of the group A Streptococcus transcriptome in experimental pharyngitis in cynomolgus macaques. Proc Natl Acad Sci U S A. 2005;102(25):9014-9019. 40. Shea PR, et al. Interactome analysis of longi- tudinal pharyngeal infection of cynomolgus macaques by group A Streptococcus. Proc Natl Acad Sci U S A. 2010;107(10):4693-4698.

41. Zhu L, et al. Streptococcus pyogenes genes that promote pharyngitis in primates. JCI Insight. 2020;5(11):e137686.

42. Zhu L, et al. Genome-wide screens identify group A Streptococcus surface proteins promoting female genital tract colonization and virulence. Am J Pathol. 2020;190(4):862-873.

43. Baruch M, et al. An extracellular bacterial pathogen modulates host metabolism to regulate its own sensing and proliferation. Cell. 2014;156(1-2):97-108.

44. Davies HC, et al. Effect of amino acids on steadystate growth of a group a hemolytic Streptococcus. J Bacteriol. 1965;89(2):421-427.

45. Johansson L, et al. Cathelicidin LL-37 in severe Streptococcus pyogenes soft tissue infections in humans. Infect Immun. 2008;76(8):3399-3404.

46. Presicce P, et al. IL-1 signaling mediates intrauterine inflammation and chorio-decidua neutrophil recruitment and activation. JCI Insight. 2018;3(6):e98306.

47. Rohde M, Cleary PP. Adhesion and invasion of Streptococcus pyogenes into host cells and clinical relevance of intracellular streptococci. In: Ferretti JJ, Stevens DL, Fischetti VA, eds. Streptococcus pyogenes: Basic Biology to Clinical Manifestations. University of Oklahoma Health Sciences Center; 2016: 1-39.

48. Thulin P, et al. Viable Group A Streptococci in macrophages during acute soft tissue infection. PLoS Med. 2006;3(3):e53.

49. Medina E, et al. Survival of Streptococcus pyogenes within host phagocytic cells: a pathogenic mechanism for persistence and systemic invasion. J Infect Dis. 2003;187(4):597-603.

50. O'Neill AM, et al. Cytosolic replication of Group A Streptococcus in human macrophages. MBio. 2016;7(2):e00020-16.

51. Flaherty RA, et al. Streptolysin S promotes programmed cell death and enhances inflammatory signaling in epithelial keratinocytes during Group A Streptococcus infection. Infect Immun. 2015;83(10):4118-4133.

52. Valderrama JA, Nizet V. Group A Streptococcus encounters with host macrophages. Future Microbiol. 2018;13(1):119-134.

53. Griffith JW, et al. Chemokines and chemokine receptors: positioning cells for host defense and immunity. Annu Rev Immunol. 2014;32(1):659-702.

54. Boldenow E, et al. Antimicrobial peptide response to group B Streptococcus in human extraplacental membranes in culture. Placenta. 2013;34(6):480-485.

55. Cohen R, et al. Screening asymptomatic households for Streptococcus pyogenes pharyngeal carriage as a part of in-hospital investigation of puerperal sepsis. Am J Infect Control. 2019;47(12):1493-1499.

56. Longo M, et al. Complete genome sequence of Streptococcus pyogenes emm28 strain M28PF1, responsible of a puerperal fever. Genome Announc. 2015;3(4):e00750-15.

57. Graham MR, et al. Virulence control in Group A Streptococcus by a two-component gene regu- 
latory system: global expression profiling and in vivo infection modeling. Proc Natl Acad Sci U S A. 2002;99(21):13855-13860.

58 . Köller T, et al. Typing of the pilus-proteinencoding FCT region and biofilm formation as novel parameters in epidemiological investigations of Streptococcus pyogenes isolates from various infection sites. J Med Microbiol. 2010;59(pt 4):442-452.

59. Ikebe $\mathrm{T}$, et al. Highly frequent mutations in negative regulators of multiple virulence genes in Group A Streptococcal toxic shock syndrome isolates. PLoS Pathog. 2010;6(4):e1000832. 60. Biswas I, et al. High-efficiency gene inactivation and replacement system for gram-positive bacteria. J Bacteriol. 1993;175(11):3628-3635.

61. Clarebout G, Leclercq R. Fluorescence assay for studying the ability of macrolides to induce production of ribosomal methylase. Antimicrob Agents Chemother. 2002;46(7):2269-2272.

62. Buscetta $\mathrm{M}$, et al. FbsC, a novel fibrinogenbinding protein, promotes Streptococcus agalactiae-host cell interactions. J Biol Chem. 2014;289(30):21003-21015.
63. Caparon MG, Scott JR. Genetic manipulation of pathogenic streptococci. Methods Enzymol. 1991;204:556-586.

64. Six A, et al. Srr2, a multifaceted adhesin expressed by ST-17 hypervirulent Group B Streptococcus involved in binding to both fibrinogen and plasminogen. Mol Microbiol. 2015;97(6):1209-1222.

65. Marcellin L et al. Endometriosis also affects the decidua in contact with the fetal membranes during pregnancy. Hum Reprod. 2015;30(2):392-405 